\author{
Julie Sarah Benbenishty \\ Jordan R. Hannink
}

\section{Non-verbal communication to restore patient-provider trust}

Received: 16 February 2015

Accepted: 19 February 2015

Published online: 20 March 2015

(C) Springer-Verlag Berlin Heidelberg and ESICM 2015

J. S. Benbenishty (匹)

Hadassah Medical Center, Jerusalem, Israel

e-mail: julie@hadassah.org.il

J. R. Hannink

Wellesley College, Wellesley, MA, USA

JH was rushed into the trauma unit after being crushed between two buses. Her injuries included pelvic fractures, thigh degloving, and third degree burn of her left leg. During her ICU hospitalization, she had a pulmonary embolism and a mesenteric event that resulted in surgery.

Communication was complicated because $\mathrm{JH}$, a young American tourist, had limited knowledge of the local tongue, and the care providers had limited English. Ventilated and alone, JH relied on non-verbal strategies as her primary form of communication during her ICU stay.

Communication psychologists often rely on a 55/38/7 formula: $55 \%$ body language, $38 \%$ voice tone, and $7 \%$ the actual words spoken.

Non-verbal communication helps to build trust which has been described as:

- A belief that our good will be taken care of

- An attitude bound to time and space in which one relies with confidence on someone

- A willingness to engage oneself in a relationship with an acceptance that vulnerability may arise

Ancient Biblical and Greek literature reveals links between emotion and facial expression as readable elements of language. Psychological studies in the 1970s and 1980s revealed that facial expressions across cultures are recognized as corresponding to six definite emotions, namely happiness, sadness, surprise, disgust, anger, and fear, and possibly other emotions such as interest and shame. The components of non-verbal communication are body posture, the act of listening, touch, facial expression, body language, mimic gestures, and feelings.

Body posture indicates interest or disinterest in another person. In nursing, posture is most important in performing active listening. However, more than medical information is transmitted when a patient or family member speaks to a nurse; often, the act of being heard can be spirit-healing for patients and family members. Thus, the health practitioner as a listener must deliberately commit to the act of listening. Using this body language creates a safe environment for the patient to speak freely, which gives practitioners insight into the patient's internal life and the patient an opportunity to begin emotional healing.

JH recounts: "Before my parents arrived to ICU, I was very alone. I was too nervous to sleep, and I couldn't verbally communicate. One of the nurses entered my room, began speaking in English, shampooing my hair. Not only did she wash my hair, which was caked with blood, she massaged my scalp gently combing through my dreads, carefully working out all of the knots. Her touch reassured me and put me at ease." This anecdote introduces the power of touch in decreasing anxiety in patients.

Sheldon writes, "the use of touch is the universal language of caring." Touch in health care has two modes: (1) touch during interventions, (2) conveying emotional messages. Since touch is required of most nurses during patient care, a nurse must retain awareness of what message her touch conveys. If a nurse is perceived as aggressive, a patient may feel unsafe. The effects are detrimental to both parties; the nurse does not receive information useful to aiding the patient's healing, and the 
patient does not feel in a safe environment and therefore vulnerable.

In the second mode, touch is used with the purpose of conveying emotional response-reassurance, understanding, and comfort, among others.

Non-verbal communication can also be intentional, as many nurses employ hand gestures to communicate with patients without a shared language. According to Finke, nurses mimic gestures and feelings, like pain, or thirst, or hunger, with hands and facial expression. They then read the patient's response, e.g. yes or no via shaking of the head or hands.

JH reports: "Being a patient, I had a broken body but also a broken soul. I had fallen into a position where I was no longer in control of my body; I was limited to bed, unable to speak. My sense for how people touched and looked at me became increasingly sensitive, because that was the extent of communication I had."

For trauma patients, guilt about the conditions that led them to ICU puts them in a state of emotional (and physical) vulnerability; "bodies and souls are broken" emphasizes JH. Nurses are responsible for creating the environment for a patient to heal. This is a space that has to be safe and nurturing, with the practitioner acting as a confident guide through the healing process, a translator between doctors, families, and patients, and a cushion for brokenness to rest upon.

The second tenement of establishing trust is the nature of communication. Patients are able to detect if nonverbal communication is genuine or robotic, if the nonverbal signals offered do not match the words being said.

JH recounts: "After a surgery, I needed staples removed. The nurse and I hadn't established a positive relationship. Trying to be reassuring, she said 'no, it won't hurt very much.' The first few staples she pulled out left me in pain, and I refused to continue. Later, a nurse I had a positive relationship with came to remove the remaining staples. I asked again if it would hurt. She stood next to my head, looked in my eyes with a look of understanding said she '[would] be as gentle as possible,' placing her hand on my forehead. She didn't lie to me, knowing I already knew the pain of having staples removed. But when she told me she would be as gentle as possible paired with her facial expression and reassuring touch, I believed she would try to make it as pain free as possible."

In this case, the trust in the nurse and the genuine response of working to not cause pain led to patient compliancy.

The effects of trust built on non-verbal communication are patients compliant with treatment, patient outcomes improve, patients are free to heal comfortably in a safe space. "I had many surgeries, and after each surgery, a doctor told me that JB, a nurse I had developed a relationship with, stood at my head during each surgery, stroking my hair. I don't remember the actual event, [but] hearing that a human being was with me throughout the surgery confirmed for me her investment in my wellbeing, not just technical health outcomes. Her investment in me made me feel I should invest more in myself, which was difficult for me," tearfully explains JH. "JB is responsible for saving my mind and my spirit."

Establishing trust with patients through communication-non-verbal and verbal-is paramount to working with patients' towards complete recovery-to become holistic medical successes. 